\title{
Sensing and control of the advanced LIGO optical configuration
}

Osamu Miyakawa, Seiji Kawamura, Benjamin Abbott, Rolf Bork, Peter Fritschel, et al.

Osamu Miyakawa, Seiji Kawamura, Benjamin Abbott, Rolf Bork, Peter Fritschel, Lisa Goggin, Jay Heefner, Alexander Ivanov, Fumiko Kawazoe, Conor Mow-Lowry, Alexei Ourjoumtsev, Sihori Sakata, Michael Smith, Kenneth A. Strain, Robert Taylor, Dennis Ugolini, Stephen Vass, Robert Ward, Alan Weinstein, "Sensing and control of the advanced LIGO optical configuration," Proc. SPIE 5500, Gravitational Wave and Particle Astrophysics Detectors, (29 September 2004); doi: 10.1117/12.552406

Event: SPIE Astronomical Telescopes + Instrumentation, 2004, Glasgow, United Kingdom 


\title{
Sensing and control of the Advanced LIGO optical configuration
}

\author{
Osamu Miyakawa ${ }^{*}$, Seiji Kawamura ${ }^{b}$, Benjamin Abbott ${ }^{a}$, Rolf Bork ${ }^{a}$, Peter Fritschel ${ }^{c}$, Lisa \\ Goggin $^{a}$, Jay Heefner ${ }^{a}$, Alexander Ivanov ${ }^{a}$, Fumiko Kawazoe ${ }^{d}$, Conor Mow-Lowry ${ }^{e}$, Alexei \\ Ourjoumtsev $^{f}$, Sihori Sakata ${ }^{d}$, Michael Smith ${ }^{a}$, Kenneth Strain ${ }^{g}$, Rober Taylor ${ }^{a}$, Dennis \\ Ugolini $^{h}$, Stephen $\operatorname{Vass}^{a}$, Robert Ward ${ }^{a}$ and Alan Weinstein ${ }^{a}$ \\ ${ }^{a}$ LIGO Laboratory, California Institute of Technology, Pasadena, CA 91125, USA; \\ ${ }^{b}$ National Astronomical Observatory of Japan, Mitaka, Tokyo 181-8588, Japan; \\ ${ }^{c}$ Department of Physics and Center for Space Research, Massachusetts Institute of Technology, \\ Cambridge, MA 02139, USA; \\ ${ }^{d}$ Department of Physics, Ochanomizu University, Bunkyo, Tokyo 112-8610, Japan; \\ ${ }^{e}$ Department of Physics, Faculty of Science, Australian National University, ACT 0200, \\ Australia; \\ fDépartement de Physique, École Normale Supérieure, 75005 Paris, France ; \\ ${ }^{9}$ Department of Physics and Astronomy, University of Glasgow, G12 8QQ, Glasgow, UK; \\ ${ }^{h}$ Department of Physics and Astronomy, Trinity University, San Antonio, TX 78212-7200, USA
}

\begin{abstract}
The LIGO Laboratory $40 \mathrm{~m}$ prototype interferometer at Caltech is being commissioned to prototype an optical configuration for Advanced LIGO. This optical configuration has to control five length degrees of freedom, and its control topology will be significantly more complicated than any other present interferometers. This paper explains the method of sensing, controls and lock acquisition.
\end{abstract}

Keywords: gravitational waves, interferometer, optics, controls, servos

\section{INTRODUCTION}

Advanced LIGO $^{1-3}$ (AdvLIGO) is a next generation gravitational wave detector which will employ a more complex optical configuration and control scheme than present detectors like LIGO, ${ }^{4}$ VIRGO, ${ }^{5}$ GEO,${ }^{6}$ TAMA. $^{7}$ The current scheme calls for a detuned cavity in addition to the Initial LIGO power recycled Fabry-Perot Michelson configuration, in order to increase the bandwidth of the detector in the presence of higher finesse Fabry-Perot arms. This configuration is called detuned resonant sideband extraction ${ }^{8}$ (DRSE), and requires the sensing and control of five length degrees of freedom of 7 suspended optics. This optical configuration is being tried on Caltech's 40 meter prototype gravitational wave interferometer ${ }^{9}(40 \mathrm{~m})$.

\subsection{The 40 meter prototype interferometer}

The $40 \mathrm{~m}$ interferometer is an engineering prototype for the Advanced LIGO optical configuration. The $40 \mathrm{~m}$ has power recycling and DRSE, in order to optimize the frequency-dependent response of the AdvLIGO interferometer, in the presence of seismic and thermal noise. Length control signals are derived from frontal modulation of the beam followed by demodulation at the three exit ports. The $40 \mathrm{~m}$ prototype is designed to have the same cavity finesses as AdvLIGO, despite the shorter cavity lengths. That is, it is optimized for AdvLIGO, not for $40 \mathrm{~m}$. The $40 \mathrm{~m}$ prototype is also a test interferometer for an output mode cleaner and a DC readout scheme which are required techniques in AdvLIGO to allow us to overcome the standard quantum limit.

Figure 1 shows the anticipated sensing performance and other important contributors to the noise for the $40 \mathrm{~m}$ prototype interferometer. It is predicted that two dips can be seen on the quantum-limited optical sensing noise

*miyakawa_o@ligo.caltech.edu; phone: 1626 395-2129; fax 1626 304-9834; ligo.caltech.edu 


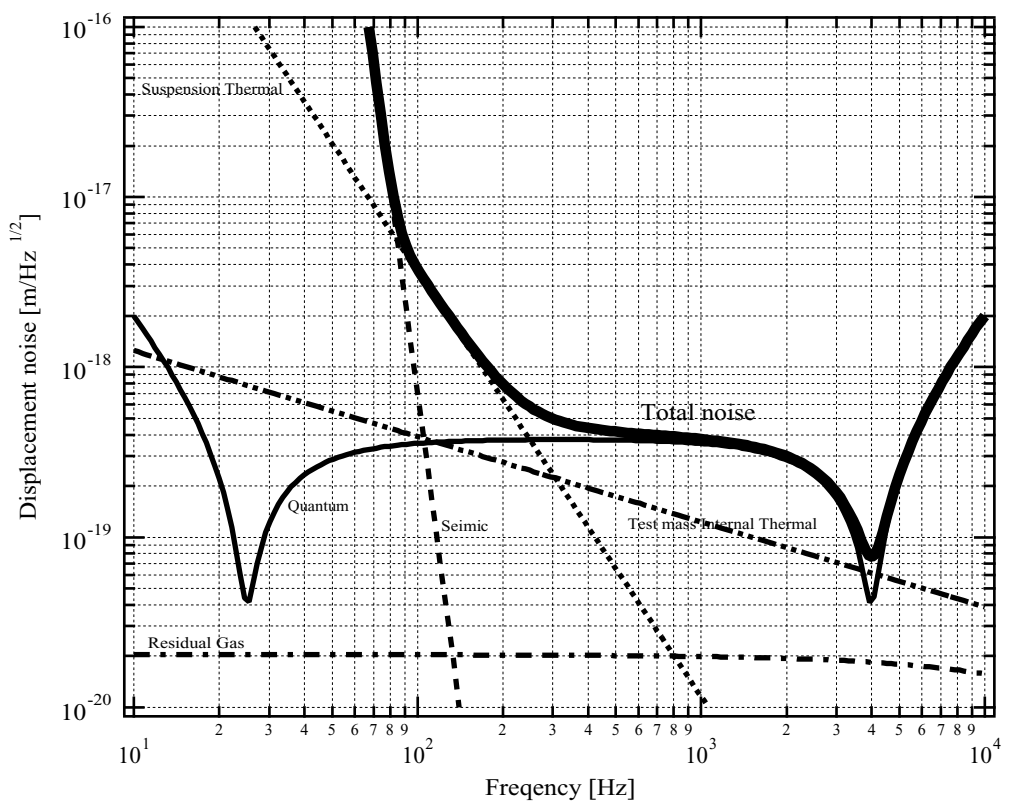

Figure 1. Target optical sensing and other significant contributions to the noise spectrum of the $40 \mathrm{~m}$ DRSE prototype interferometer.

(labeled as Quantum in the figure) because of the DRSE configuration. These dips can overcome the standard quantum limit at certain frequencies. To see these dips is one of the purposes of $40 \mathrm{~m}$ prototype. Unfortunately, the lower frequency dip is obscured by other noise sources making it impossible to see directly, but it may be seen as a optical spring effect ${ }^{10-12}$ when the transfer function of the interferometer is measured.

\subsection{Difference between Advanced LIGO and $40 \mathrm{~m}$ prototype interferometer}

There are some other significant differences between $40 \mathrm{~m}$ and AdvLIGO. From the viewpoint of the optical configuration, the cavity length of the $40 \mathrm{~m}$ is $1 / 100$, and the finesse is the same as AdvLIGO. This means that the storage time of the light in the arm cavities becomes 1/100 which may result in significant differences for the lock acquisition dynamics. The modulation frequencies chosen for the $40 \mathrm{~m}$ of $33 \mathrm{MHz}$ and $166 \mathrm{MHz}$ are different from those intended for AdvLIGO, $9 \mathrm{MHz}$ and $180 \mathrm{MHz}$, so that the signal diagonalization may be worse. Thermal effects which will be a problem in AdvLIGO are negligible because the $40 \mathrm{~m}$ uses a LIGO-like laser with $6 \mathrm{~W}$ incident on the interferometer, while AdvLIGO will use $125 \mathrm{~W}$.

The $40 \mathrm{~m}$ uses LIGO-like single pendulums to suspend mirrors, while AdvLIGO will use quadruple pendulums to suspend mirrors. For low frequency seismic attenuation, the $40 \mathrm{~m}$ uses commercial active seismic isolation system called STACIS which has isolation of up to $25 \mathrm{~dB}$ in the $1-100 \mathrm{~Hz}$ band. This is inferior to the AdvLIGO active seismic isolation under development. ${ }^{13,14}$ Moreover, the $40 \mathrm{~m}$ is located in the Los Angeles metropolitan area, so that the influence of the ground motion is much larger than at the AdvLIGO sites.

The $40 \mathrm{~m}$ employs a digital control system, and we digitize all length control signals at $16384 \mathrm{~Hz}$, and send digital signals to the digital suspension controllers to move the suspended mirrors. This sampling rate is the same as the sampling rate of LIGO's digital control system. This limits a unity gain frequency of the control to typically $300 \mathrm{~Hz}$ because of the time delay of the digital part. We are not sure that this will be fast enough for AdvLIGO because the finesse of AdvLIGO is more than 20 times higher than the finesse of Initial LIGO. 


\section{SIGNAL EXTRACTION FOR LENGTH SENSING}

The $40 \mathrm{~m}$ DRSE consists of two input test masses (ITMx and ITMy), two end test masses (ETMx and ETMy), a beam splitter (BS), a power recycling mirror (PRM), and a signal extraction mirror (SEM) illuminated by a laser beam as shown in Fig. 2 . It has five length degrees of freedom (DOFs) to control: $L_{+}, L_{-}, l_{+}, l_{-}$, and $l_{\mathrm{s}}$ as defined in Fig. 2. There are three optical ports, where we can obtain the length signals from: a symmetric port (SP), an asymmetric port (AP), and a pick-off port (PO).

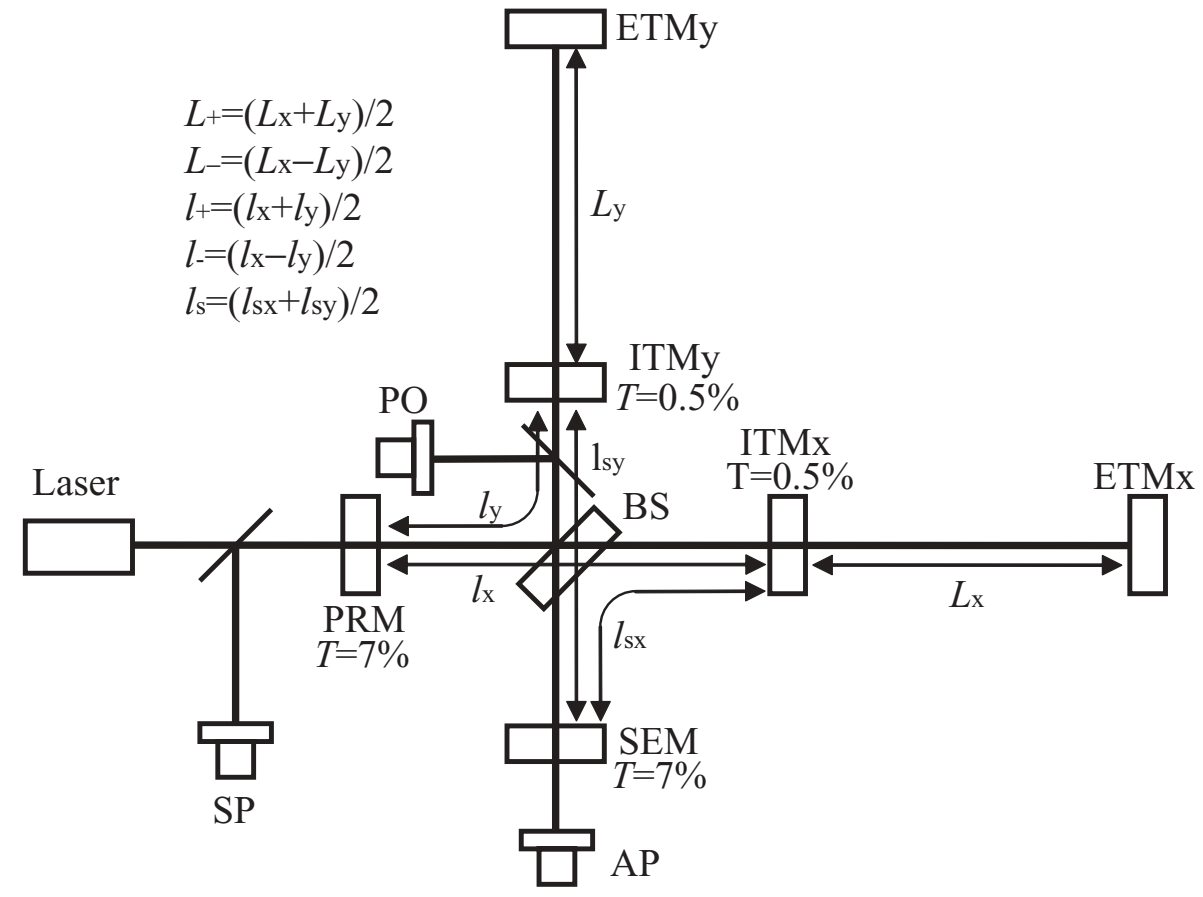

Figure 2. Schematic diagram of the $40 \mathrm{~m}$ DRSE interferometer.

Control signals derived from beats between carrier and sidebands are dominated by the arm length variations, because of the high gain in the high finesse arms. Therefore, we choose to derive signals for the short, lower finesse cavity lengths $\left(l_{+}, l_{-}, l_{s}\right)$ using beats between sidebands only, so as to be insensitive to arm length variations $\left(L_{+}, L_{-}\right)$. Double demodulation (DDM) allows us to extract signals from the beats between sidebands that are nicely diagonal.

The laser light is phase-modulated at two different frequencies: $f_{1}=33.207 \mathrm{MHz}$ on $\mathrm{EOM}_{1}$ and $f_{2}=166.033 \mathrm{MHz}$ on $\mathrm{EOM}_{2}$ as shown in Fig. 3. This allows us to obtain length signals using single demodulation at $f_{1}, f_{2}$, or differential modulation at $f_{2} \pm f_{1}$, and double demodulation at a combination of $f_{2}-f_{1}$ and $f_{2}+f_{1}\left(f_{1} \times f_{2}\right)$. The Michelson macroscopic asymmetry is set in such a way that the $+f_{2}$ sideband incident to the BS is completely transmitted to the asymmetric port of the BS when the carrier incident on the BS is completely reflected back to the symmetric BS port. The macroscopic length of the power recycling cavity (PRC) is set in such a way that all the sidebands, both $\pm f_{1}$ and $\pm f_{2}$ are resonant when the carrier is anti-resonant in the PRC by itself. Note that the carrier becomes resonant in the PRC cavity when the carrier is resonant in the arm cavities because of the phase flipping due to the arm cavities (See lower left of Fig. 3.). The macroscopic length of the signal extraction cavity (SEC) is set in such a way that the $+f_{2}$ sideband is exactly anti-resonant when the carrier is appropriately detuned from resonance in the SEC (it is this detuning which produces the desired dips in the quantum-limited optical sensing noise seen in Fig. 1). Also note that the phase of the $+f_{2}$ sideband is advanced by $\pi / 2$ when it is transmitted from one BS port to the other BS port due to the macroscopic Michelson asymmetry, thus the round-trip phase advance due to this effect is $\pi$ (See lower right of Fig. 3.). These conditions make the $+f_{2}$ sideband resonant in the combined cavity consisting of the PRC and SEC. On the other hand the $\pm f_{1}$ sidebands 


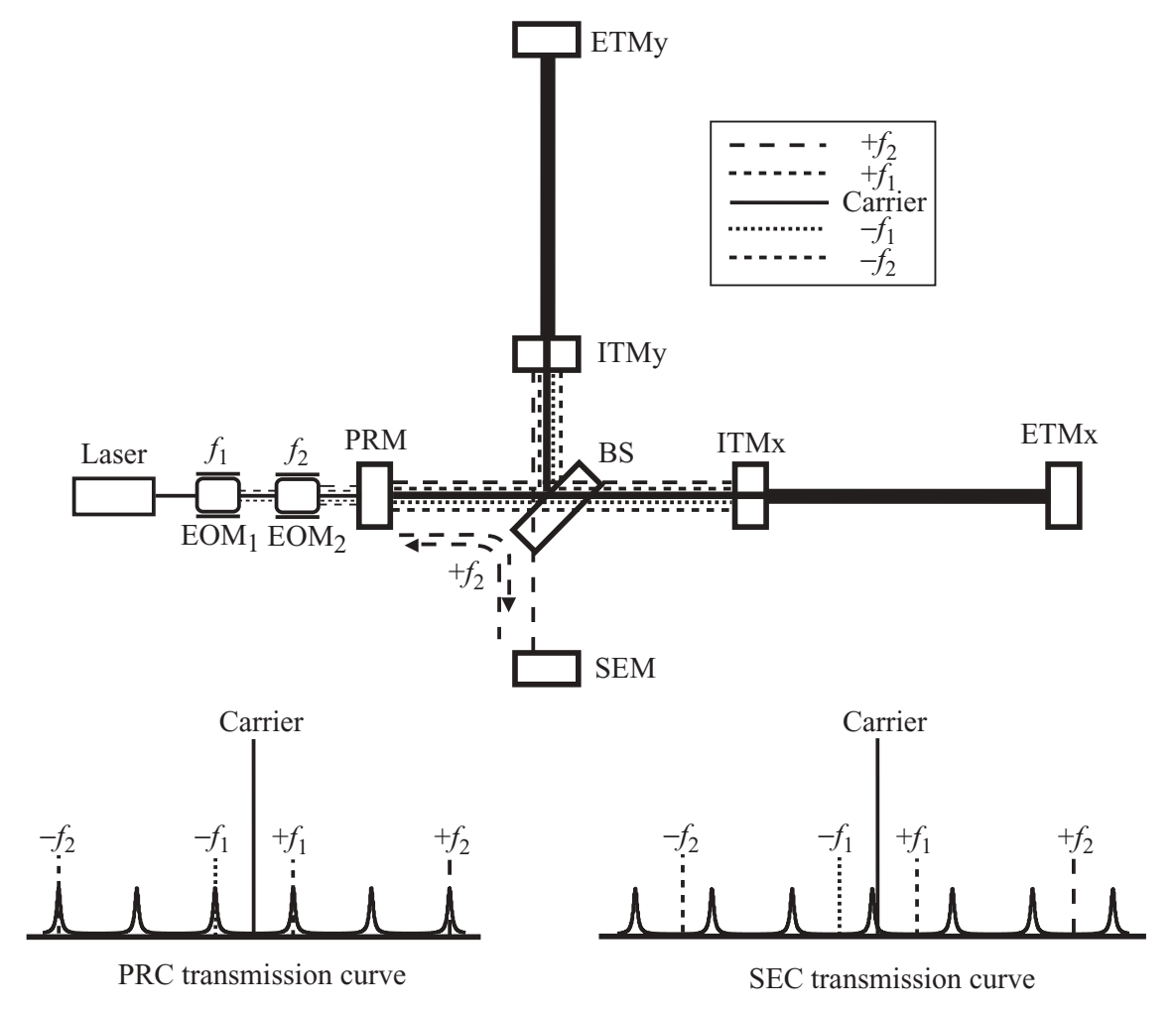

Figure 3. Two phase modulations and macroscopic optical configuration.

are resonant only in the PRC and leak into the SEC only slightly. This scheme can be brought over to AdvLIGO with minor modifications: $f_{1}=9 \mathrm{MHz}, f_{2}=180 \mathrm{MHz}$, to account for the longer PRC and SEC lengths at the sites.

Two EOMs placed in series produce sidebands impressed on sidebands on the laser light and cause the DDM signals to contain undesired beats between carrier and sidebands. Control signals for $l_{+}, l_{-}, l_{s}$ are therefore disturbed by the carrier resonance in the PRC, SEC and long arm cavities and will significantly degrade the signal diagonalization. (See Appendix A.). This effect of the sidebands of sidebands was not taken into account when the optical configuration of AdvLIGO and $40 \mathrm{~m}$ was originally designed.

After some further analysis and tabletop prototyping, we will adopt a method to eliminate the sidebands-onsidebands, and our discussion below assumes this situation.

\section{SIGNAL SENSING}

\subsection{Double demodulation}

The demodulation phases for the double demodulation are optimized to remove the DC offset and maximize the desired length signal. Here the contour plots for the $l_{+}, l_{-}$, and $l_{s}$ signals and DC offset at the SP are shown as an example in Fig. 4.

To optimize the demodulation phases, one has to find a pair of demodulation phases that lie on the dotted thick line $(\mathrm{DC}=0)$ and are also close to the top (or bottom) of the desired signal contour. The question is whether that point is close to zero for the other DOFs. From the figures, it can be seen that such a point exists, and the best signal for $l_{+}$is shown as triangle. 


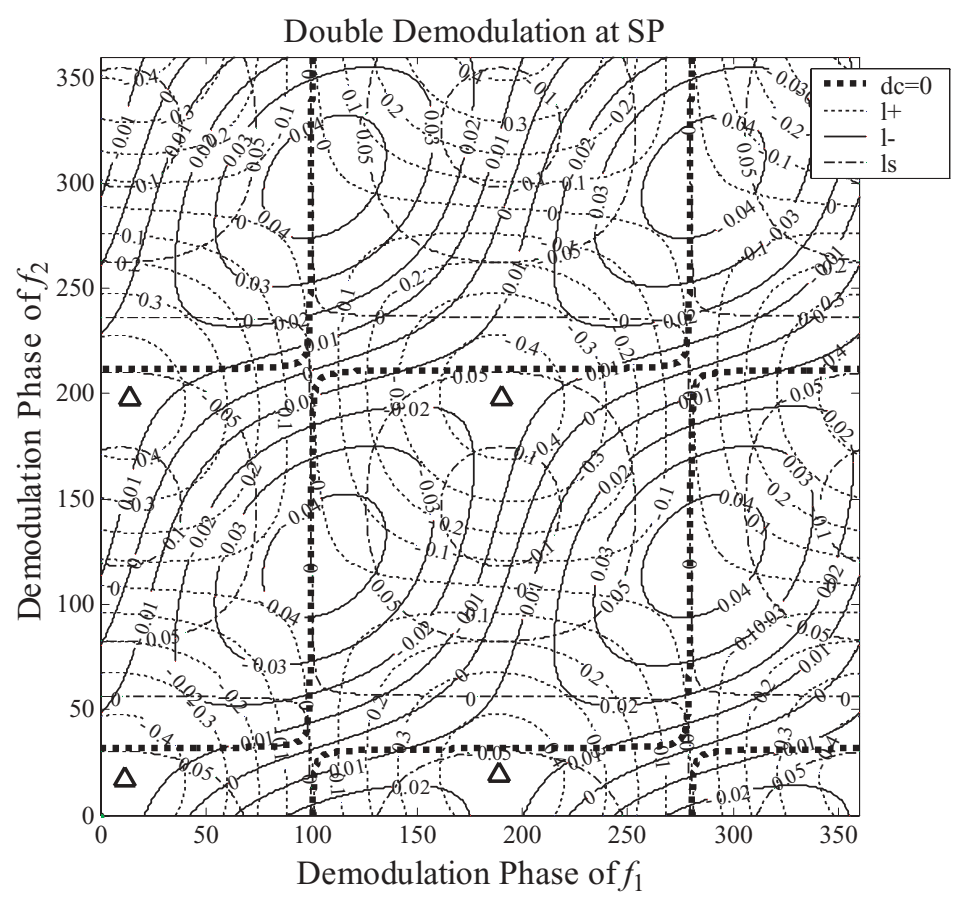

Figure 4. Dependence of the signals and DC offset at SP on the demodulation phases. Triangle shows the best point for $l_{+}$signal.

\subsection{Signal matrix}

Table 1 shows the matrix for 5 length degrees of freedom. Here the demodulation phases are optimized to remove DC offsets and to maximize the desired length signal.

Table 1. Signal matrix with the ideal cavity length.

\begin{tabular}{|l|l|l|r|r|r|r|r|}
\hline Port & Dem. freq. & Dem. phase & $L_{+}$ & $L_{-}$ & $l_{+}$ & $l_{-}$ & $l_{s}$ \\
\hline SP & $f_{1}$ & 10 & 1 & $-3.8 \mathrm{E}-9$ & $-1.2 \mathrm{E}-3$ & $-1.3 \mathrm{E}-6$ & $-2.3 \mathrm{E}-6$ \\
\hline AP & $f_{2}$ & 271 & $-4.8 \mathrm{E}-9$ & 1 & $-1.2 \mathrm{E}-8$ & $-1.3 \mathrm{E}-3$ & $-1.7 \mathrm{E}-8$ \\
\hline SP & $f_{1} \times f_{2}$ & 189,32 & $-1.7 \mathrm{E}-3$ & $-3.0 \mathrm{E}-4$ & 1 & $-3.2 \mathrm{E}-2$ & $-1.0 \mathrm{E}-1$ \\
\hline AP & $f_{1} \times f_{2}$ & 4,81 & $-6.2 \mathrm{E}-4$ & $1.5 \mathrm{E}-3$ & $7.5 \mathrm{E}-3$ & 1 & $7.1 \mathrm{E}-2$ \\
\hline PO & $f_{1} \times f_{2}$ & 164,12 & $3.6 \mathrm{E}-3$ & $2.7 \mathrm{E}-3$ & $4.6 \mathrm{E}-1$ & $-2.3 \mathrm{E}-2$ & 1 \\
\hline
\end{tabular}

The original design of AdvLIGO and $40 \mathrm{~m}$ made use of differential demodulation to extract the $l_{+}$and $l_{-}$signal. Since double demodulation has two adjustable parameters (demodulation phases) while differential demodulation has only one, it is possible to produce a better signal matrix with double demodulation. Our circuit design for the photo detector and demodulation system allows both double demodulation and differential demodulation (we can switch from one to the other just by changing a relevant parameter in a real-time control computer), it is advantageous to use double demodulation for all the three length signals, $l_{+}, l_{-}$, and $l_{\mathrm{s}}$.

\subsection{Acceptable tolerance for cavity length}

An important question is how the signal matrix is affected by the deviation of the macroscopic cavity length from the design length. 
Table 2. Signal matrix with $l_{+}$of $1 \mathrm{~cm}$ tolerance.

\begin{tabular}{|l|l|l|r|r|r|r|r|}
\hline Port & Dem. freq. & Dem. phase & $L_{+}$ & $L_{-}$ & $l_{+}$ & $l_{-}$ & $l_{s}$ \\
\hline SP & $f_{1}$ & 334 & 1 & $-7.6 \mathrm{E}-9$ & $-1.2 \mathrm{E}-3$ & $-4.1 \mathrm{E}-6$ & $-2.3 \mathrm{E}-6$ \\
\hline AP & $f_{2}$ & 230 & $-1.3 \mathrm{E}-9$ & 1 & $-3.0 \mathrm{E}-8$ & $-1.3 \mathrm{E}-3$ & $-1.7 \mathrm{E}-8$ \\
\hline SP & $f_{1} \times f_{2}$ & 162,73 & $-6.5 \mathrm{E}-4$ & $-3.5 \mathrm{E}-4$ & 1 & $-5.6 \mathrm{E}-2$ & $-1.3 \mathrm{E}-1$ \\
\hline AP & $f_{1} \times f_{2}$ & 173,218 & $1.5 \mathrm{E}-3$ & $4.2 \mathrm{E}-4$ & -2.1 & 1 & $-2.4 \mathrm{E}-1$ \\
\hline PO & $f_{1} \times f_{2}$ & 329,153 & $1.1 \mathrm{E}-3$ & $2.7 \mathrm{E}-3$ & 2.6 & $-1.6 \mathrm{E}-1$ & 1 \\
\hline
\end{tabular}

The matrix with an $l_{+}$length deviation of $1 \mathrm{~cm}$ is shown in Table 2 as an example of tolerance. Here the demodulation phases for the double demodulation are optimized for each length deviation, and the demodulation phase for single demodulation is optimized to maximize the desired length signal. A cross coupling of greater than unity in the normalized matrix is not desirable. From similar calculations, the acceptable cavity length tolerances are approximately $6 \mathrm{~cm}$ for $l_{-}$and $3 \mathrm{~mm}$ for $l_{+}$and $l_{s}$.

Based on these calculations, the actual cavity lengths in the $40 \mathrm{~m}$ interferometer have been adjusted within $\pm 1 \mathrm{~mm}$ error. The actual signal matrix is thereby expected to contain off-diagonal terms that are all significantly less than 1.

\section{LOCK ACQUISITION}

The lock acquisition strategy we take here is very simple: we lock one DOF at a time until we lock all the five DOFs. We lock the first DOF, and it has to be robust even though the other four DOFs are free. Then we lock the second DOF, and it has to be robust even though the other three DOFs are free. We continue locking the remaining DOFs just one by one until all the DOFs are locked.

This approach is very different from the approach taken for the lock acquisition of the initial LIGO, where although each lock is not robust enough the next lock is accomplished with the aid of lock acquisition software quickly before the first lock is lost. We believe that the approach presented here is more suitable for the more complex AdvLIGO optical configuration, especially in the R\&D phase. The only question is whether or not such well-behaved signals can exist.

In order to realize this strategy we first divide the lock acquisition procedure of the whole interferometer into the following two stages:

1. Lock the central part of the interferometer consisting of the ITMs, BS, PRM, and SEM using the beat signal between $f_{1}$ and $f_{2}$, while blocking the long arm cavities;

2. Lock the arm cavities consisting of the ITMs and ETMs using beats between carrier and $f_{1}$ or $f_{2}$.

We expect that the lock of the central part of the interferometer can be maintained when the arm cavities are locked. If the carrier were used to lock the central part, the polarity of the control signal would be flipped when the arm cavities are locked. Thus we could lose the lock of the central part. The sidebands are not resonant in the arm cavities, however. This means that the amplitude and polarity of the signals obtained only from the sidebands do not depend on whether the arm cavities are locked or not. Therefore the lock of the central part would not be disturbed when the arm cavities are locked.

We expect that the lock of the central part is robust even if the arm cavities are freely swinging. The worry is that occasional flashing of the sidebands in the arm cavities will vary the amplitude and maybe even the polarity of the signals obtained from the sidebands, which potentially will make the central part to fall out of lock. Nevertheless we think that the disturbances are probably too fast to cause loss of lock thanks to the high finesse of the arm cavities. We are also encouraged by the fact that TAMA300 demonstrated that the lock of the 
central part ${ }^{15}$ (a power recycled Michelson) with the signals obtained from the third harmonic demodulation ${ }^{16}$ (beat between the first-order sideband and the negative-second-order sideband) was robust even when the arm cavities were freely swinging.

The lock of the central part can be attempted with both arm cavities blocked. In this way we can concentrate on the lock of the central part without any disturbances from the arm cavities. Once the central part is locked only with the sidebands we plan to unblock the arm cavities to see if the lock of the central part is maintained.

The lock of the central part can be acquired by the following steps:

1. Lock $l_{-}$using a dither signal (as a temporary lock acquisition signal)

2. Lock $l_{+}$using a DDM signal at the SP

3. Lock $l_{s}$ using a DDM signal at the PO port

4. Switch the $l_{-}$control signal from the dither signal to the DDM signal at the AP

The reason why the dither signal is introduced a temporary intermediate step in the lock acquisition process is because we found that no DDM signals are robust enough to maintain the lock of the first DOF while the other two DOFs are freely swinging. Even the best DDM signal $\left(l_{+}\right)$gives a sensible signal for only $80 \%$ of the 2 -dimensional space of possible microscopic lengths of $l_{-}$and $l_{s}$. The polarity of the signal will be flipped from time to time depending on $l_{-}$and $l_{s}$, which makes it impossible to maintain the lock with this signal.

\section{1. $l_{-}$lock with dither signal}

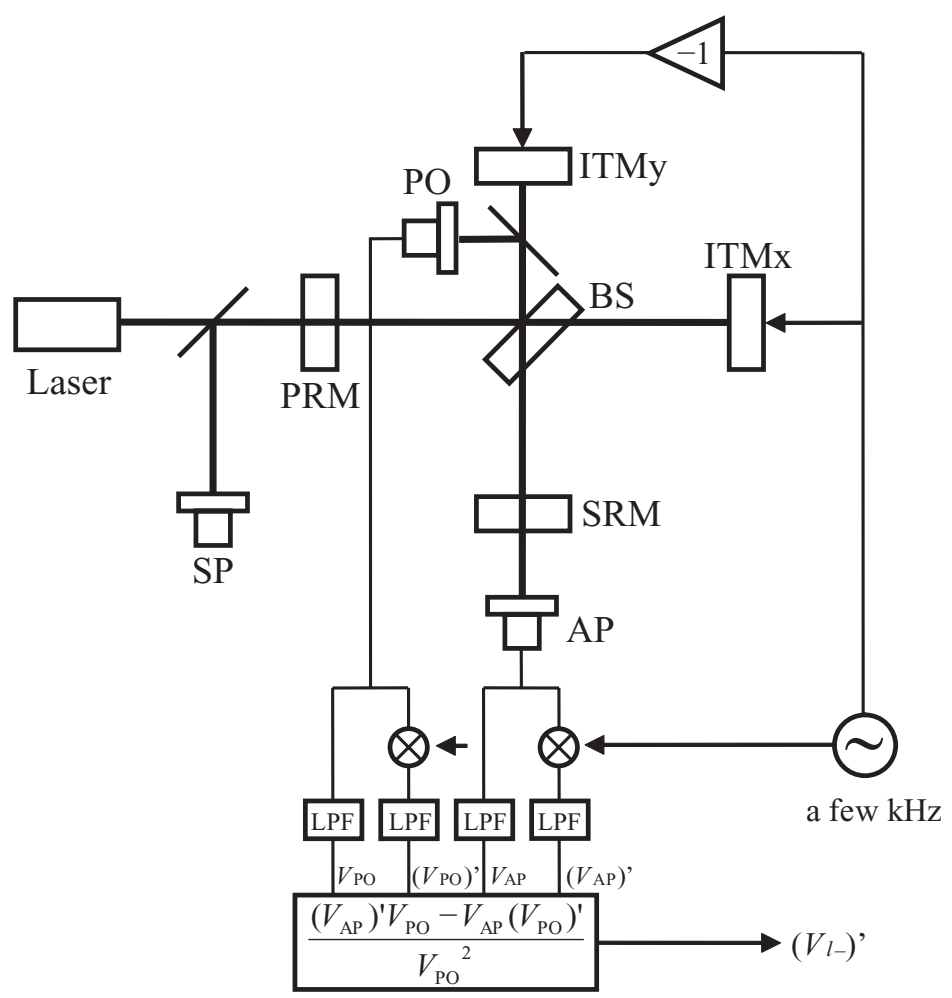

Figure 5. $l_{-}$signal obtained by the dither method.

The error signal to lock $l_{-}$is obtained by the dither method. We shake the two ITMs differentially at a few kHz. As shown in Fig. 5, we detect the light power at the PO and AP: $V_{\mathrm{PO}}$ and $V_{\mathrm{AP}}$, and demodulate the 
signals with the dither frequency to obtain $\left(V_{\mathrm{PO}}\right)^{\prime}$ and $\left(V_{\mathrm{AP}}\right)^{\prime}$. We calculate the following error signal in the front-end length sensing computer,

$$
\left(V_{l_{-}}\right)^{\prime}=\frac{\left(V_{\mathrm{AP}}\right)^{\prime} V_{\mathrm{PO}}-V_{\mathrm{AP}}\left(V_{\mathrm{PO}}\right)^{\prime}}{V_{\mathrm{PO}}^{2}} .
$$

This signal is the derivative of the following signal with respect to $l_{-}$

$$
V_{l_{-}}=\frac{V_{\mathrm{AP}}}{V_{\mathrm{PO}}} .
$$

Note that all the signals, $V_{\mathrm{PO}}, V_{\mathrm{AP}},\left(V_{\mathrm{PO}}\right)^{\prime}$, and $\left(V_{\mathrm{AP}}\right)^{\prime}$ are low-pass-filtered to remove the components at the dither frequency before doing the math.

It was found that $V_{l_{-}}$does not depend on $l_{+}$at all but only depends on $l_{s}$. This can be explained by the consideration that the light power at PO and AP is affected by the displacement of the PRM in the same manner; thus they are cancelled when divided.

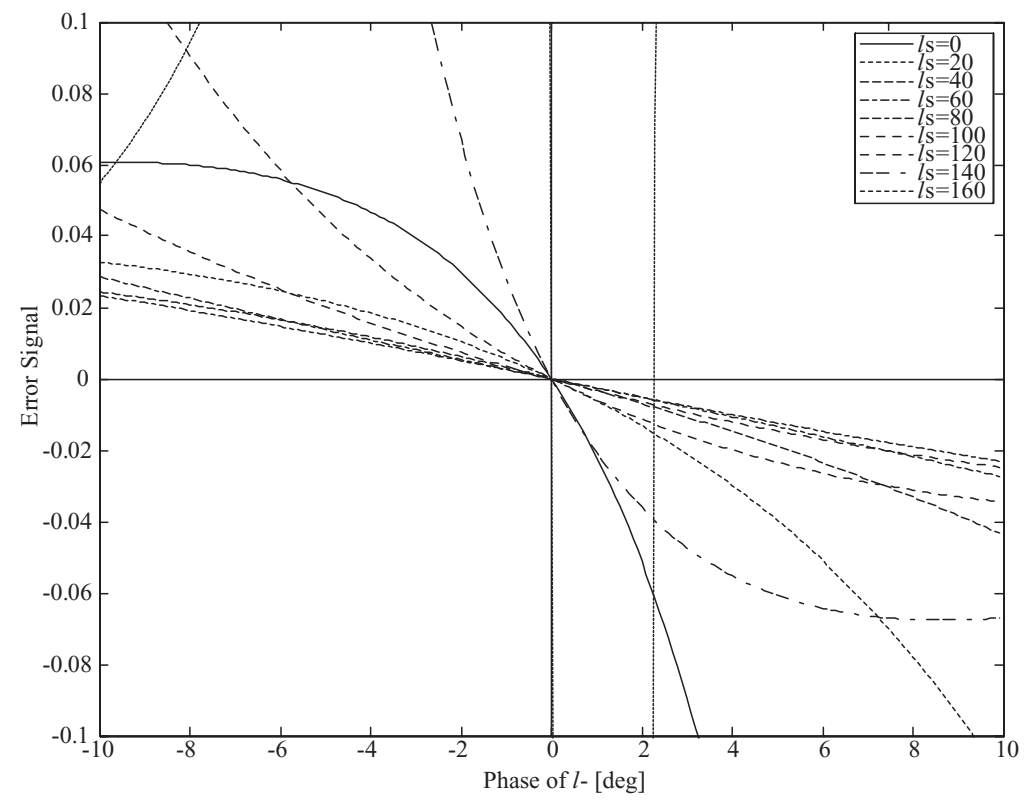

Figure 6. Dependence of the $\left(V_{l_{-}}\right)^{\prime}$ signal on $l_{s}$.

The advantage of this signal is that its slope around $l_{-}=0$ is of the same polarity for any $l_{s}$ as shown in Fig. 6. Note that the $\left(V_{l_{-}}\right)^{\prime}$ signal also crosses zero with a slope of the same polarity at $l_{-}=90^{\circ}$, which is not a desirable locking point. Therefore with a chance of $50 \%$, we can lock to the undesirable point.

Also note that this signal has a huge error signal around some undesirable phase of $l_{-}$depending on $l_{s}$. Fortunately, however, the slope of this signal is opposite to the slope of the desirable error signal. Thus it can never be locked to those points with a proper polarity of the servo system, although we plan to limit the signal range to remove the disturbances of those undesirable error signals.

Although the slope of the $\left(V_{l_{-}}\right)^{\prime}$ signal has the same polarity for any $l_{s}$, the slope of the error signal varies significantly depending on $l_{s}$. The servo system for the $l_{-}$lock has to be designed in such a way that the system is as stable as possible with this varying control loop gain. Nevertheless the system would probably experience an oscillation for $l_{s}$ that gives too high slope. We still expect that the oscillation happens in such a short period that it will not cause unlocking of the system. If the oscillation causes the system to fall out lock, we plan to implement a limiter that prevents a large oscillation. 
Another concern of the $\left(V_{l_{-}}\right)^{\prime}$ signal is that the signal quality is degraded by the existence of the RF modulations. The disturbances are not so crucial with a modulation depth of 0.01 , but significantly worse with a modulation depth of 0.03 . To obtain a clean signal, we plan to turn off the RF modulations first. After $l_{-}$ is locked we plan to gradually increase the modulation depth to see how much modulation depth we can have without kicking the system out of lock and to see if that modulation depth is large enough to obtain sensible signals for the next steps.

\section{2. $l_{+}$lock with double demodulation}
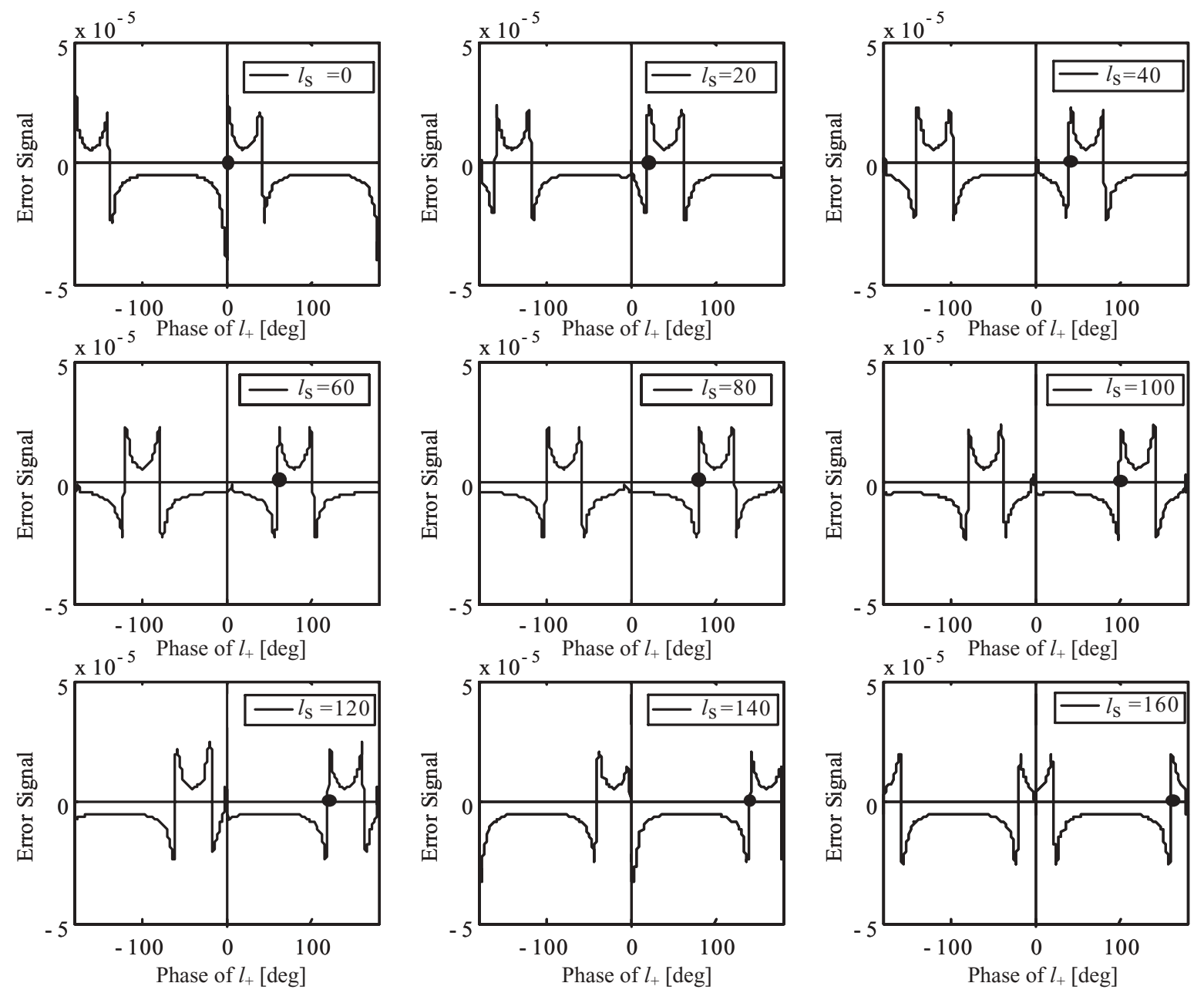

Figure 7. Dependence of the DDM signal for $l_{+}$on $l_{s}$. The DDM phases are optimized for the most symmetric signal curve.

From now on we assume that $l_{-}$is always locked to the ideal point. As the second step the error signal to lock $l_{+}$is obtained by DDM at the SP. For the final operation, the DDM phases have to be chosen to minimize the offset and to maximize the desirable signal. This is not the case, however, for the lock acquisition of $l_{+}$.

Fortunately, we found that the DDM error signal for $l_{+}$behaves well when the DDM phases are optimized for the most symmetric dependence as shown in Fig. 7 . Note that the zero-crossing for $l_{+}$depends on $l_{s}$. That is, the lock point of $l_{+}$exactly follows the displacement of $l_{s}$. This is because this error signal is given by the condition that $+f_{2}$ sideband is resonant in the PRC+SEC combined cavity. When the $l_{-}$is locked to the ideal point, the carrier incident to the BS is all reflected back to the PRM, whereas the $+f_{2}$ sideband is all sent to the 
SEM. The $+f_{2}$ sideband is then reflected by the SEM, and sent to the PRM this time. Therefore the combined cavity for the $+f_{2}$ sideband practically becomes a simple cavity consisting of the PRM and SEM. Thus the PRM will exactly follow the motion of the SEM in order to keep the resonance condition of $+f_{2}$.

\section{3. $l_{s}$ lock with double demodulation}

From now on we assume that $l_{-}$is always locked to the ideal point and $l_{+}$is following the motion of $l_{s}$. As the third step the error signal to lock $l_{s}$ is obtained by the DDM at PO. Here the DDM phases are chosen to minimize the offset and maximize the $l_{s}$ signal. The obtained error signal is reasonable as shown in Fig. 8 .

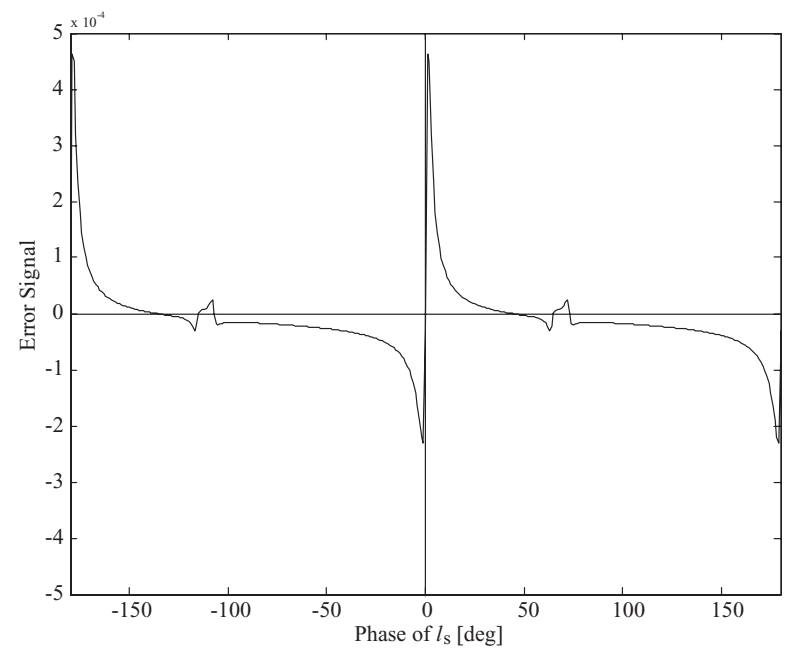

Figure 8. DDM signal for $l_{s}$. The DDM phases are optimized for no offset and maximum $l_{s}$.

\subsection{Lock acquisition of arm cavities}

As mentioned above, the lock of the central part of the interferometer is expected to be stable if there are no sidebands of sidebands because the resonance of the carrier in the arms would not disturb the central part. When the central part is stable, we can lock the arm degrees of freedom using light at the SP and AP to control the $L_{+}$ and $L_{-}$degrees of freedom. The gravitational wave signal can be derived from the $L_{-}$sensing or control signals.

\section{SUMMARY AND CHALLENGES}

We discussed the detuned resonant sideband extraction optical configuration for Advanced LIGO using the $40 \mathrm{~m}$ prototype interferometer. Double demodulation is useful to diagonalize the signal matrix with five degrees of freedom if no sidebands of sidebands exist. Also lock acquisition is promising using dither locking for $l_{-}$, double demodulation for $l_{+}$when $l_{-}$is at the ideal point and double demodulation for $l_{s}$ when $l_{-}$is at the ideal point and $l_{+}$is resonant at $f_{2}$. Lock acquisition of the arm cavities is also promising if no sidebands of sidebands exist.

We have prepared for all suspended mirrors and circuits including the double demodulation except for the method to realize operation with no sidebands on sidebands. The Mach-Zehnder interferometer as one of the methods will be ready soon and we expect to achieve the first lock of a suspended detuned resonant sideband extraction interferometer with power recycling.

\section{APPENDIX A. EFFECT OF SIDEBANDS OF SIDEBANDS}

In our baseline design, the various modulations are added via sequential EOMs. We found that it is necessary to include the effect of sidebands impressed on sidebands by these EOMs to correctly predict the DC offsets and the signal matrix. We describe this complication in this section. 


\section{A.1. Less offset on signal}

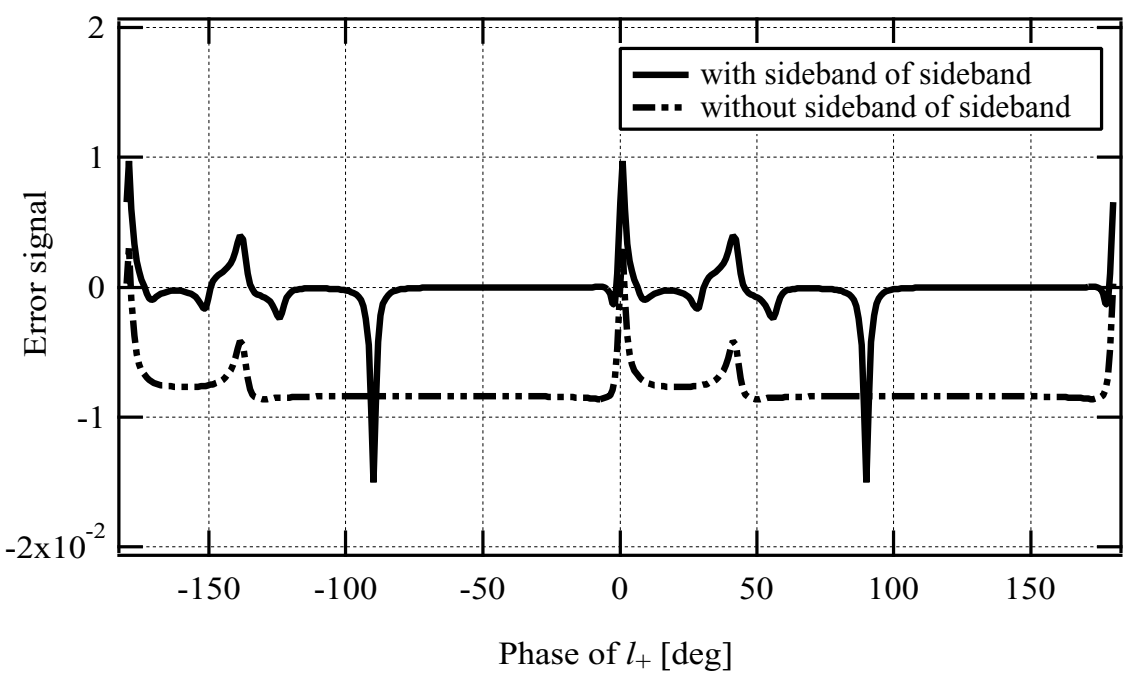

Figure 9. Error signal of $l_{+}$when $l_{-}$and $l_{s}$ is at ideal point with/without sidebands of sidebands.

A big difference occurs in the response of interferometer at low frequency when the sidebands of sidebands are taken into account in the calculation. ${ }^{17}$ Fig. 9 shows the $l_{+}$signal when the sidebands of sidebands do not exist and do exist in the calculation. It can be seen that the sidebands of sidebands has the effect of removing the offset from the signal. If there are no sidebands of sidebands, the phase modulation of phase modulation by two series EOMs has a component of amplitude modulation, and will result in an offset when it is demodulated at the frequency of the sidebands of sidebands. It may be thought that the signal with the sidebands of sidebands seems to be easier to work with and looks to be of higher quality for control. However, from the view point of the diagonalization of signal, it will be much worse.

\section{A.2. Beat signals between carrier and $f_{2} \pm f_{1}$}

The double demodulation can be considered as a sum of the demodulation of $f_{2}+f_{1}$ and the demodulation of $f_{2}-f_{1}$. Actually, the double demodulation of $40 \mathrm{~m}$ has been achieved in exactly this way. If the sidebands of sidebands exist in $f_{2}+f_{1}$ and $f_{2}-f_{1}$, the beat signals between carrier and $f_{2} \pm f_{1}$ also can be mixed into desired signal(See Fig. 10). These signals are -2 order and they are the same order as desired signal which is extracted without the sidebands of sidebands. The signal of the double demodulation is disturbed by the carrier resonance.

Table 3 shows a signal matrix when the sidebands of sidebands is considered.

Table 3. Signal matrix with sidebands of sidebands.

\begin{tabular}{|l|l|l|r|r|r|r|r|}
\hline Port & Dem. freq. & Dem. phase & $L_{+}$ & $L_{-}$ & $l_{+}$ & $l_{-}$ & $l_{s}$ \\
\hline SP & $f_{1}$ & 10 & 1 & $-1.4 \mathrm{E}-8$ & $-1.2 \mathrm{E}-3$ & $-1.4 \mathrm{E}-6$ & $-6.2 \mathrm{E}-6$ \\
\hline AP & $f_{2}$ & 271 & $1.2 \mathrm{E}-7$ & 1 & $1.4 \mathrm{E}-5$ & $1.3 \mathrm{E}-3$ & $6.5 \mathrm{E}-6$ \\
\hline SP & $f_{1} \times f_{2}$ & 184,30 & 7.4 & $-3.4 \mathrm{E}-4$ & 1 & $-3.3 \mathrm{E}-2$ & $-1.1 \mathrm{E}-1$ \\
\hline AP & $f_{1} \times f_{2}$ & 4,73 & $-5.7 \mathrm{E}-4$ & 32 & $7.1 \mathrm{E}-1$ & 1 & $7.1 \mathrm{E}-2$ \\
\hline PO & $f_{1} \times f_{2}$ & 161,4 & 3.3 & 1.7 & $1.9 \mathrm{E}-1$ & $-3.5 \mathrm{E}-2$ & 1 \\
\hline
\end{tabular}



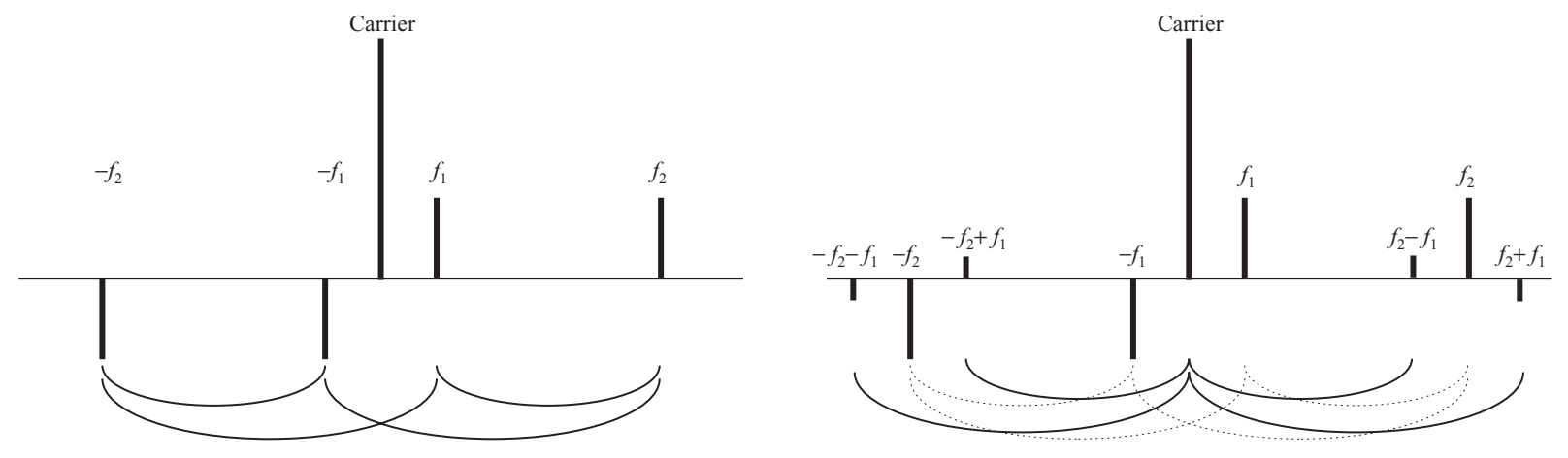

Figure 10. No sidebands of sidebands exist in the light (left) and sidebands of sidebands exist in the light (right). The double demodulation signal has components of the beat signal between carrier and $f_{2} \pm f_{1}$ if sidebands of sidebands exist. Relationship of up and down means phase modulation and relationship of up and up or down and down means amplitude modulation.

Diagonalization of the signal matrix is very difficult as shown in this table and it also disturbs the lock acquisition. The reason is that the central part (including Michelson, PRC and SEC) may be knocked out of lock by the flashing of the carrier in the arm cavities when the arms are moving freely. Even if the arms are blocked, lock acquisition of the central part may be difficult because of carrier resonances within the PRC or SEC.

\section{A.3. Avoiding sidebands of sidebands}

We can address this complication in several ways. The first idea is to place the EOMs in parallel in a MachZehnder interferometer. Sidebands of sidebands are not generated from parallel EOMs because sidebands $f_{1}$ and $f_{2}$ are generated only from the carrier and then they are summed up. However, in this setup it is necessary to control one more mirror to lock the Mach-Zehnder interferometer. The second idea is to cancel out $f_{2} \pm f_{1}$ using two more EOMs. In this case, a monitor for $f_{2} \pm f_{1}$ is needed (such as an optical spectrum analyzer) to adjust amplitudes and phases carefully. Another idea is to apply a voltage which can cancel $f_{2} \pm f_{1}$ electrically on existing EOMs. However, with the resonant type EOMs used in $40 \mathrm{~m}$, high voltage signals are needed to produce enough $f_{2} \pm f_{1}$ signals outwith the range of EOM resonance. In this case, a monitor as mentioned above is also needed. We plan to implement a Mach-Zender interferometer to ensure that no sidebands of sidebands are generated.

\section{ACKNOWLEDGMENTS}

This work is supported by the National Science Foundation cooperative agreement PHY0107417. This document has been assigned LIGO Laboratory document number LIGO-P040017-00-R. We thank the many members of the LIGO Laboratory, the LIGO Scientific Collaboration and the engineering team especially J. Romie, H. Armandula and G. Billingsley. We also thank to A. Freise for developing the FINESSE simulation tool.

\section{REFERENCES}

1. E. Gustafson, D. Shoemaker, K. Strain, and R. Weiss, "LSC white paper on detector research and development," LIGO Document Number T990080-00-D, 1999.

2. "LIGO II conceptual project book," LIGO Document Number L990267-00-M, 1999.

3. P. Fritschel, "Second generation instruments for the Laser Interferometer Gravitational Wave Observatory (LIGO)", Gravitational-Wave Detection, M.Cruise, P. Saulson Eds. 4856, p. 282-291, SPIE, Bellingham, WA. 2003

4. D. Sigg, "Commissioning of the LIGO detectors," Class. Quantum, Grav. 19, p. 1429, 2002. 
5. F. Acernese et al, "The present status of the VIRGO Central Interferometer," Class. Quantum, Grav. 19, p. 1421, 2002.

6. B Willke et al, "The GEO 600 gravitational wave detector," Class. Quantum, Grav. 19, p. 1377, 2002.

7. M. Ando and the TAMA Collaboration, "Current status of TAMA," Class. Quantum, Grav. 19, p. 1409, 2002.

8. J. Mizuno, "Resonant sideband extraction: a new configuration for interferometric gravitational wave detectors," Phys. Lett. A 175, pp. 273-276, 1993.

9. A. Weinstein, "Advanced LIGO optical configuration and prototyping effort," Class. Quantum, Grav. 19, p. $1575,2002$.

10. A. Buononno and Y. Chen, "Optical noise correlations and beating the standard quantum limit in advanced gravitational-wave detectors," Class. Quantum, Grav. 18, p. L95, 2001.

11. A. Buononno and Y. Chen, "Quantum noise in second generation, signal-recycled laser interferometric gravitational-wave detectors," Phys. Rev. D 64, p. 042006, 2001.

12. A. Buononno and Y. Chen, "Signal recycled laser-interferometer gravitational-wave detectors as optical springs," Phys. Rev. D 65, p. 042001, 2002.

13. R. Abbott, R. Adhikari, G. Allen, S. Cowley, E. Daw, D. DeBra, J. Giaime, G. Hammond, M. Hammond, C. Hardham, J. How, W. Hua, W. Johnson, B. Lantz, K. Mason, R. Mittleman, J. Nichol, S. Richman, J. Rollins, D. Shoemaker, G. Stapfer, and R. Stebbins, "Seismic isolation for advanced ligo," Class. Quantum Grav. 19, p. 1591, 2002.

14. N. A. Robertson, G. Cagnoli, D. R. M. Crooks, E. Elliffe, J. E. Faller, P. Fritschel, S. Gosler, A. Grant, A. Heptonstall, J. Hough, H. Luck, R. Mittleman, M. Perreur-Lloyd, M. V. Plissi, S. Rowan, D. H. Shoemaker, P. H. Sneddon, K. A. Strain, C. I. Torrie, H. Ward, and P. Willems, "Seismic isolation for advanced ligo," Class. Quantum Grav. 19, p. 4043, 2002.

15. K. Arai and the TAMA Collaboration, "Sensing and controls for power-recycling of TAMA300," Class. Quantum Grav. 19, p. 1843, 2002.

16. K. Arai, M. Ando, S. Moriwaki, K. Kawabe, and K. Tsubono, "New signal extraction scheme with harmonic demodulation for power-recycled Fabry-Perot-Michelson interferometers," Phys. Lett A, 273, p. 15, 2000.

17. K. Kawabe, "An information note on FINESSE: Sidebands of sidebands, http://www.rzg.mpg.de/ adf/," 2002 . 